\title{
AHP ANSWERS TO PROBLEMS WITH KNOWN COMPOSITE VALUES
}

\author{
William C. Wedley ${ }^{1}$ \\ Faculty of Business Administration \\ Simon Fraser University \\ Burnaby, B. C. Canada, V5A 1S6 \\ wedley@sfu.ca
}

Keywords: AHP measurement modes, ratio answers

Summary: A problem with a known composite answer is used to test six different AHP modes: distributive, ideal, referenced, linking pins, benchmark and supermatrix. All except the ideal mode give the correct composite answer. Upon deletion of an alternative, all except the distributive mode maintain the original ratios. The appropriateness of the various modes is discussed.

\section{Introduction}

At the 5th International Symposium of the Analytic Hierarchy Process in Kobe Japan, Thomas Saaty suggested to the author that the controversy regarding correct synthesis modes for the AHP should be tested with problems with known true values. If the decomposed problem can be synthesized to produce the known composite value, then that fact is an indication that the synthesis is correct.

The use of problems with known answers has been utilized before, particularly for the validation of the eigenvector routine. For example, the eigenvector solution has been tested with the measuring distances from Philadelphia, intensities of lights and areas of objects (Saaty, 1977, Saaty \& Vargas, 1991). In essence, these situations are single criterion problems. For multiple criteria situations, validation with the use of problems with known answers has received less attention. Although Schoner and Wedley (1989) used a car purchase example and Wedley et al $(1993,1996)$ used multiple distances from Singapore, these applications have been criticized as being reducible to a single criterion (Forman, 1993; Hauser and Peniwati, 1996). Nevertheless, Vargas (1997) used a known answer problem to show that multiplicative composition gives rise to invalid answers. Similarly, Saaty (1999) uses known purchase prices and remodeling costs of houses to show that multiplicative synthesis does not yield the correct composite ratio.

This short treatise utilizes a problem with a known answer to test various methods of additive synthesis. The chosen modes to be tested are the distributive and ideal modes from conventional AHP, referenced AHP, linking pins AHP, benchmark measurement and the supermatrix. The selected problem with known answers is Vargas' (1997) example with 3 boxes with 4 objects that were used to show that the multiplicative AHP produces invalid results. Here, we use the same example in a similar manner to test various methods of deriving AHP composite priorities. Like Vargas, we choose the ability of each method to replicate the true composite ratios as the measure of effectiveness. Ratio preservation rather than rank preservation is chosen as the measure of effectiveness, because AHP produces ratio answers. With perfect accuracy, inconsistency cannot be the reason why ratios are deflected from their true values.

\footnotetext{
${ }^{1}$ Acknowledgement: The author thanks the Natural Sciences and Engineering Research Council of Canada for financial support related to this project.
} 


\section{The Problem}

Vargas's example with a known answer is shown in Table 1. Three boxes each contain different components of 4 objects. Each component has a different weight (lbs). The objective is to determine the relative composite priorities for the true total weights of the objects when the components are assembled together. These are shown in the last two columns. In decision analysis terminology, the assembled objects to be measured are the alternatives and the boxes are the component criteria.

Table 1 -- Three Boxes with Four Objects of Known Weights

\begin{tabular}{|lccc|c|c|c|}
\hline & Box 1 & Box 2 & Box 3 & Total & True weights & Ratio to Best \\
\hline Object 1 & 1 & 6 & 10 & 17 & 0.243 & 0.850 \\
Object 2 & 2 & 4 & 14 & 20 & 0.286 & 1.000 \\
Object 3 & 3 & 8 & 6 & 17 & 0.243 & 0.850 \\
Object 4 & 4 & 2 & 10 & 16 & 0.229 & 0.800 \\
\hline Total & 10 & 20 & 40 & 70 & 1 & \\
\hline
\end{tabular}

\subsection{The Distributive Mode}

The distributive mode normalizes alternative priorities to sum to one. According to Axiom 3 of AHP, the criteria are assumed to be independent of the alternatives. Here, where the objective is to measure the total weight of the objects, we follow Vargas (1997) in utilizing the relative weights of the boxes as the criteria weights. As can be seen from Table 2, the composite priorities represent the true weights.

Table 2 -- Distributive Mode with Independent Criteria Weights

\begin{tabular}{lccc|c|c|}
\hline Criteria & $10 / 70=$ & $20 / 70=$ & $40 / 70=$ & & \\
\cline { 5 - 7 } priorities= & 0.143 & 0.286 & 0.571 & Composite & Ratio to \\
\hline & Box 1 & Box 2 & Box 3 & Priorities & Best \\
\hline Object 1 & 0.1 & 0.3 & 0.25 & 0.243 & 0.850 \\
Object 2 & 0.2 & 0.2 & 0.35 & 0.286 & 1.000 \\
Object 3 & 0.3 & 0.4 & 0.15 & 0.243 & 0.850 \\
Object 4 & 0.4 & 0.1 & 0.25 & 0.229 & 0.800 \\
\hline \multicolumn{7}{c}{ Total $=$} & 1.000 & \\
\hline
\end{tabular}

\subsection{The Ideal Mode}

With the ideal mode, the best alternative under each criterion is normalized to unity. As shown in Table 3, these items are Objects 4, 3 and 2 for Boxes 1, 2 and 3, respectively. As for relative measurement, the

Table 3 -- Ideal Mode with Independent Criteria Weights

\begin{tabular}{|c|c|c|c|c|c|c|}
\hline \multirow{2}{*}{$\begin{array}{l}\text { Criteria } \\
\text { priorities= }\end{array}$} & \multirow{2}{*}{$\begin{array}{c}10 / 70= \\
0.143\end{array}$} & \multirow{2}{*}{$\begin{array}{c}20 / 70= \\
0.286\end{array}$} & \multirow{2}{*}{$\begin{array}{c}40 / 70= \\
0.571\end{array}$} & 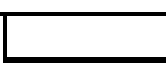 & 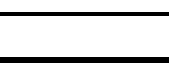 & \\
\hline & & & & Composite & Re- & Ratio to \\
\hline & Box 1 & Box 2 & Box 3 & Priorities & normalized & Best \\
\hline Object 1 & 0.250 & 0.750 & 0.714 & 0.658 & 0.243 & 0.838 \\
\hline Object 2 & 0.500 & 0.500 & 1.000 & 0.786 & 0.291 & 1.000 \\
\hline Object 3 & 0.750 & 1.000 & 0.429 & 0.638 & 0.236 & 0.812 \\
\hline Object 4 & 1.000 & 0.250 & 0.714 & 0.622 & 0.230 & 0.792 \\
\hline & & & Total = & 2.704 & 1 & \\
\hline
\end{tabular}


criteria are assumed to be independent of the alternatives and derived in the same manner as relative measurement. Although the composite priorities no longer sum to unity, they can be easily renormalized to unity if they are in ratio form. The sixth column of Table 3 shows such renormalization. The last column shows the same ratios as a fraction of the best alternative (Object 2).

The important point to note with the ideal mode is that the composite priorities, whether composite or renormalized, are not in the same ratio as was determined with relative measurement. Here, we have the unusual situation where two different versions of conventional AHP give different composite answers. Which one is correct will be addressed below.

\subsection{Referenced AHP}

Referenced AHP (Schoner and Wedley, 1989) is the same as the distributive mode, except that the criteria weights represent either the average criterion possessed by the alternatives or the total criterion possessed by all the alternatives. Except for that requirement, referenced AHP is the same as the distributive mode. Since the criteria weights used in the distributive mode example are determined by that principle, the referenced AHP results are the same as displayed in Table 2.

\subsection{The Linking Pins Mode}

Linking Pin AHP (Schoner et al, 1993) is similar to the ideal mode except for two differences: (1) any alternative, not just the best alternative, can be chosen as the link that is normalized to take $100 \%$ of the criterion weight and (2) criteria weights are established in reference to the specific alternatives chosen as links. In the linking pins procedure, the alternatives used as comparison references (linkages) when establishing criteria weights receive $100 \%$ of the criterion weights that they represent. Other alternatives then receive their commensurate weights during the aggregation process via reference to the linkages.

Table 4 -- Linking Pin Mode with Criteria Weights for the Link Elements

\begin{tabular}{|c|c|c|c|c|c|c|}
\hline \multirow{2}{*}{$\begin{array}{l}\text { Criteria } \\
\text { priorities= }\end{array}$} & \multirow{2}{*}{$\begin{array}{c}2 / 20= \\
0.1\end{array}$} & \multirow{2}{*}{$\begin{array}{c}8 / 20= \\
0.4\end{array}$} & \multirow{2}{*}{$\begin{array}{c}10 / 20= \\
0.5\end{array}$} & \multirow[b]{2}{*}{ Composite } & \multirow[b]{2}{*}{ Re- } & \multirow[b]{2}{*}{ Ratio to } \\
\hline & & & & & & \\
\hline & Box 1 & Box 2 & Box 3 & Priorities & normalized & Best \\
\hline Object 1 & 0.5 & 0.75 & 1 & 0.850 & 0.243 & 0.850 \\
\hline Object 2 & 1 & 0.5 & 1.4 & 1.000 & 0.286 & 1.000 \\
\hline Object 3 & 1.5 & 1 & 0.6 & 0.850 & 0.243 & 0.850 \\
\hline Object 4 & 2 & 0.25 & 1 & 0.800 & 0.229 & 0.800 \\
\hline & & & & 3.500 & 1 & \\
\hline
\end{tabular}

In Table 4, Objects 2, 3 and 1 have been used as the linkage elements for criteria (Boxes) 1, 2, and 3 respectively. As well, the criteria priorities represent the importance of each of those linkages. Since the sum of the linkages is $20 \mathrm{lbs}$., their correct criteria weights are 2/20, 8/20 and 10/20 respectively. As can be seen from Table 4, the composite and renormalized weights are in correct ratio form.

It should be noted that the ideal alternatives could have been used as linkages. If so, the criteria priorities for those linkages are $4 / 26,8 / 26$ and $14 / 26$, respectively. The reader should verify that putting these priorities into Table 3 with the ideal items normalized to unity would yield the correct composite weights.

\subsection{The Benchmark Mode}

In the Benchmark mode of AHP (Wedley et al., 1996), a single representative alternative or a small number of alternatives becomes the benchmark by which other alternatives are compared. If a small set of alternatives is used as the benchmark, then Referenced AHP becomes the means to determine their priorities. If a single alternative is to be used as the benchmark, then linking pin AHP is used to generate its priority. After this step is completed, each additional alternative is compared sequentially with the 
benchmarks. During composition to get each new alternative's priority, the benchmarks are normalized to unity similar to what is done in the linking pin mode.

Table 5 -- Benchmark Mode Objects 3 and 4 as Benchmarks

\begin{tabular}{|lccc|c|c|}
\hline Criteria & $7 / 33=$ & $10 / 33=$ & $16 / 33=$ & & \\
priorities & 0.212 & 0.303 & 0.485 & Composite & Ratio to \\
\hline & Box 1 & Box 2 & Box 3 & Priorities & Best \\
\hline Object 3 & 0.429 & 0.800 & 0.375 & 0.515 & 1 \\
Object 4 & 0.571 & 0.200 & 0.625 & 0.485 & 0.941 \\
\hline \multicolumn{7}{r}{ Total $=$} & 1.000 & \\
\cline { 4 - 6 }
\end{tabular}

Table 5 shows the derived priorities when Objects 3 and 4 are used as benchmarks. The criteria priorities represent the relative importance of those two objects and the resulting priorities maintain the true ratios between the objects (i.e. $.485 / 515=.941$ in Table 5 is the same as $.800 / .850=.941$ in Table 1).

Subsequent alternatives are compared to the two benchmark alternatives and take their global priorities from the benchmark values. Table 6 demonstrates the inclusion of Objects 1 and 2 as referents to the benchmarks. Notice that the alternative priorities are normalized so that the benchmarks continue to sum to one. This can be accomplished by a proportional transformation of the alternative priorities of any of the previous Tables. Notice too that the criteria priorities are unchanged with the insertion of the compared alternatives. This means that the criteria priorities remain relevant for the benchmark alternatives but not the non-benchmark alternatives. Accordingly during synthesis, the composite priorities for the benchmarks attain their true ratios and the compared alternatives take their ratios in proportion to the benchmarks. Renormalization of the composite priorities of Table 6 so that they sum to unity demonstrates that the benchmark mode achieves the true priorities shown in Table 1.

Table 6 -- Benchmark Mode Objects 3 and 4 as Benchmarks

\begin{tabular}{|c|c|c|c|c|c|c|}
\hline Criteria & $7 / 33=$ & $10 / 33=$ & $16 / 33=$ & & & \\
\hline priorities & 0.212 & 0.303 & 0.485 & Composite & Re- & Ratio to \\
\hline & Box 1 & Box 2 & Box 3 & Priorities & normalized & Best \\
\hline Benchmar & & & & & & \\
\hline Object 3 & 0.429 & 0.800 & 0.375 & 0.515 & 0.243 & 0.401 \\
\hline Object 4 & 0.571 & 0.200 & 0.625 & 0.485 & 0.229 & 0.377 \\
\hline Comparec & Alterna & & Total $=$ & 1 & & \\
\hline Object 1 & 0.143 & 0.600 & 0.625 & 0.515 & 0.243 & 0.401 \\
\hline Object 2 & 0.286 & 0.400 & 0.875 & 0.606 & 0.286 & 0.471 \\
\hline & & & Total $=$ & 1.121 & & \\
\hline
\end{tabular}

\subsection{The Supermatrix Mode}

The supermatrix mode is also known as a system with feedback. It is the general case for AHP insofar as it can handle situations of dependence and independence of the criteria on the alternatives. Local priorities are generated from the perspective of both the alternatives given the criteria and the criteria given the alternatives. 
Table 7 -- The Supermatrix, Starting State

\begin{tabular}{l|c|c|c|c|c|c|c|}
\hline \multicolumn{3}{c}{1} & Box 1 & Box 2 & Box 3 & Object 1 & \multicolumn{2}{c|}{ Object 2 Object 3 Object 4 } \\
\hline Box 1 & 0 & 0 & 0 & 0.059 & 0.100 & 0.176 & 0.250 \\
\cline { 2 - 8 } Box 2 & 0 & 0 & 0 & 0.353 & 0.200 & 0.471 & 0.125 \\
\cline { 2 - 8 } Box 3 & 0 & 0 & 0 & 0.588 & 0.700 & 0.353 & 0.625 \\
\cline { 2 - 8 } Object 1 & 0.100 & 0.300 & 0.250 & 0 & 0 & 0 & 0 \\
\cline { 2 - 8 } Object 2 & 0.200 & 0.200 & 0.350 & 0 & 0 & 0 & 0 \\
\cline { 2 - 8 } Object 3 & 0.300 & 0.400 & 0.150 & 0 & 0 & 0 & 0 \\
\cline { 2 - 8 } Object 4 & 0.400 & 0.100 & 0.250 & 0 & 0 & 0 & 0 \\
\hline
\end{tabular}

In Table 7, the values in the first 3 columns represent the priorities for the Objects, given that they are in Box 1, 2 and 3 respectively. The values in the last 4 columns represent the relative importance of each criterion (Boxes) from the perspective of a specific item (Object) in each box. These values are the starting point of the supermatrix process.

Since each column of Table 7 is columnar stochastic, the matrix can be multiplied by itself to find the effect of interactions between criteria and alternatives. When Table 7 is multiplied by itself a sufficient number of times, the values behave as a Markov process and stabilize to the values shown in Table 8 . Notice that the stabilized priorities for alternatives are the true composite priorities and that the stabilized values for the criteria represent the relative weights of the boxes when all objects are included.

Table 8 -- The Supermatrix, Stable State

\begin{tabular}{|c|c|c|c|c|c|c|c|}
\hline & Box 1 & Box 2 & Box 3 & Object & Object 2 & Object 3 & Object 4 \\
\hline \multirow{3}{*}{$\begin{array}{l}\text { Box } 1 \\
\text { Box } 2 \\
\text { Box } 3 \\
\end{array}$} & 0 & 0 & 0 & 0.143 & 0.143 & 0.143 & 0.143 \\
\hline & 0 & 0 & 0 & 0.286 & 0.286 & 0.286 & 0.286 \\
\hline & 0 & 0 & 0 & 0.571 & 0.571 & 0.571 & 0.571 \\
\hline \multirow{4}{*}{$\begin{array}{l}\text { Object } 1 \\
\text { Object } 2 \\
\text { Object } 3 \\
\text { Object } 4\end{array}$} & 0.243 & 0.243 & 0.243 & 0 & 0 & 0 & 0 \\
\hline & 0.286 & 0.286 & 0.286 & 0 & 0 & 0 & 0 \\
\hline & 0.243 & 0.243 & 0.243 & 0 & 0 & 0 & 0 \\
\hline & 0.229 & 0.229 & 0.229 & 0 & 0 & 0 & 0 \\
\hline
\end{tabular}

\section{The Effect of Additions or Deletions}

The main criticism of AHP has been the existence of rank reversal if alternatives are added or deleted. Some claim that rank reversal is natural and should be allowed, while others claim that rank reversals are symptomatic of a flaw in AHP procedures. In this paper, we do not consider reversals of rank. Instead, we concentrate on the ratio stability of priorities. The reason for this is that AHP purports to be a method for ratio measurement. Accordingly, the criterion of interest is the ability of AHP to produce correct ratios. Our test is to see what happens to relative ratios when an item is added or deleted.

Instead of adding an alternative, we have chosen to remove Object 1 from each box. Object 1 was chosen for removal because it is not the ideal alternative for any criterion and because it has the same overall priority as Object 3. With it removed, the remaining Objects have true priority weights that are

Table 9 -- Three Boxes with Object 1 Removed

\begin{tabular}{lccc|c|c|c|}
\hline & Box 1 & Box 2 & Box 3 & Total & True weights & Ratio to Best \\
\hline Object 2 & 2 & 4 & 14 & 20 & 0.377 & 1.000 \\
Object 3 & 3 & 8 & 6 & 17 & 0.321 & 0.850 \\
Object 4 & 4 & 2 & 10 & 16 & 0.302 & 0.800 \\
\hline Total & 9 & 14 & 30 & 53 & 1 & \\
\hline
\end{tabular}


differentiated from one another. This makes it easier to understand the dynamics of AHP. The correct weights and composite priorities with Object 1 removed are shown in Table 9.

\subsection{The Distributive Mode with Object 1 Removed}

The distributive mode answer, with Object 1 removed is presented in Table 10. As is usual for this mode, we have left criteria priorities unchanged on the assumption that they are independent of the alternatives. As before, the rank of the priorities remains unchanged (Object $2>$ Object $3>$ Object 4 ). The ratios, however, have not. Whereas Object 3 was formerly $.243 / 286=.850$ of Object 2 it is now.325/.380 $=.856$ of Object 2. Similarly, Object 4 is now .776 of Object 2 whereas it was formerly .800. Accordingly, the distributive mode fails the ratio stability test.

Table 10 -- Distributive Mode with Object 1 Removed

\begin{tabular}{|c|c|c|c|c|c|}
\hline \multirow{3}{*}{$\begin{array}{l}\text { Criteria } \\
\text { priorities= }\end{array}$} & \multicolumn{3}{|c|}{$10 / 70=20 / 70=40 / 70=$} & \multirow[b]{2}{*}{ Composite } & \multirow[b]{2}{*}{ Ratio } \\
\hline & 0.143 & & 0.571 & & \\
\hline & Box 1 & Box 2 & Box 3 & Priorities & to Best \\
\hline Object 2 & 0.222 & 0.286 & 0.467 & 0.380 & 1.000 \\
\hline Object 3 & 0.333 & 0.571 & 0.200 & 0.325 & 0.856 \\
\hline \multirow[t]{2}{*}{ Object 4} & 0.444 & 0.143 & 0.333 & 0.295 & 0.776 \\
\hline & & & & 1.000 & \\
\hline
\end{tabular}

\subsection{The Ideal Mode with Object 1 Removed}

Table 11 shows the ideal mode with Object 1 removed. As compared to the previous ideal solution shown in Table 3, there is no change in the ratio of one priority to another. Thus, the ideal mode maintains rank and ratio relationships provided the ideal does not change. Nevertheless, we should observe that the ideal mode again fails to reproduce the true priorities shown in Table 9

Table 11 -- Ideal Mode with Object 1 Removed.

\begin{tabular}{|c|c|c|c|c|c|c|}
\hline \multirow{2}{*}{$\begin{array}{l}\text { Criteria } \\
\text { priorities= }\end{array}$} & \multicolumn{6}{|c|}{$10 / 70=20 / 70=40 / 70=$} \\
\hline & 0.143 & 0.286 & 0.571 & Composite & Re- & Ratio \\
\hline & Box 1 & Box 2 & Box 3 & Priorities & normalized & to Best \\
\hline Object 2 & 0.5 & 0.5 & 1 & 0.786 & 0.384 & 1.000 \\
\hline ect 3 & 0.75 & 1 & 0.429 & 0.638 & 0.312 & 0.812 \\
\hline Object 4 & 1 & 0.25 & 0.714 & 0.622 & 0.304 & 0.792 \\
\hline & & & Total $=$ & 2.046 & 1 & \\
\hline
\end{tabular}

\subsection{Referenced AHP with Object 1 Removed}

In Referenced AHP, criteria priorities are determined in reference to the alternatives in the choice set. Since Object 1 has been removed, these weights have changed. The results with the new weights are shown in Table 12. Notice that Referenced AHP reproduces the true composite weights with no change in rank or ratios. 
Table 12 -- Referenced AHP with Object 1 Removed

\begin{tabular}{|c|c|c|c|c|c|}
\hline \multirow{2}{*}{$\begin{array}{l}\text { Criteria } \\
\text { priorities= }\end{array}$} & $9 / 53$ & $14 / 53$ & $30 / 53$ & & \\
\hline & 0.170 & 0.264 & 0.566 & Composite & Ratio \\
\hline & Box 1 & Box 2 & Box 3 & Priorities & to Best \\
\hline Object 2 & 0.222 & 0.286 & 0.467 & 0.377 & 1.000 \\
\hline Object 3 & 0.333 & 0.571 & 0.200 & 0.321 & 0.850 \\
\hline Object 4 & 0.444 & 0.143 & 0.333 & 0.302 & 0.800 \\
\hline & & & Total $=$ & 1 & 2.65 \\
\hline
\end{tabular}

\subsection{The Linking Pins Mode with Object 1 Removed}

Table 13 shows the linking pin mode with Object 2 used as the link for each criterion. Since Object 1 was a link in the previous example, it was necessary to choose a new link element for Criterion 3 . For illustrative purposes, we have chosen to show the same object as link for all three criteria. As was done in Table 4, different objects could have been chosen as the link for each criterion. Whatever links are used, the criteria weights reflect the weight of those links. In Table 13, the criteria weights represent the importance of Object 2.

Table 13 -- Linking Pin Mode with Object 1 Removed

\begin{tabular}{|lccc|c|c|c|}
\hline Criteria & $2 / 20=$ & $4 / 20=$ & $14 / 20=$ & & & \\
priorities= & 0.100 & 0.200 & 0.700 & Composite & Re- & Ratio \\
\hline & Box 1 & Box 2 & Box 3 & Priorities & normalized & to Best \\
\hline Object 2 & 1 & 1 & 1 & 1.000 & 0.377 & 1.000 \\
Object 3 & 1.5 & 2 & 0.429 & 0.850 & 0.321 & 0.850 \\
Object 4 & 2 & 0.5 & 0.714 & 0.800 & 0.302 & 0.800 \\
\hline
\end{tabular}

As can be seen from Table 13, the linking pin mode maintains ratio relationships irrespective of the alternative or alternatives used as links.

\subsection{The Benchmark Mode with Object 1 Removed}

In the benchmark mode, each new alternative is compared to the composite result of the benchmark alternatives. In other words, non-benchmark alternatives are independent of each other and are only dependent upon the benchmark alternatives. So long as the benchmarks are not changed or removed, there will be no impact upon the addition or deletion of other alternatives.

When Object 1 is removed from Table 6, the effect is the same as deleting that row. The priorities of the benchmarks (Objects 3 and 4 ) and the other non-benchmark (Object 2) remain unchanged. Hence, the benchmark mode maintains ratio relationships upon deletion or addition on non-benchmarks.

\subsection{The Supermatrix Mode with Object 1 Removed}

With Object 1 removed, the starting matrix for the Supermatrix mode is given in Table 14. After matrix multiplication, the stable state is given in Table 15. As can be seen, the supermatrix mode generates the correct composite weights shown in Table 9.

Notice in the supermatrix that the starting criteria weights given Object 2 (Table 14) are the same as the linking pin weights in Table 13 (which also produces the correct composite weights). Had Object 3 or 4 been used as the common link in Table 13, the same correct composite weights could have been achieved with different linkages. This implies that the starting criteria for the supermatrix correspond to linking pin 
criteria with a common linkage. The difference between the two methods is that linking pins establishes the weights through hierarchical composition whereas the supermatrix does it via matrix multiplication.

Notice too that the stabilized criteria weights in Table 15 are the same as those used in Referenced AHP (Table 12). This implies that the supermatrix's stabilized weights for criteria represent the average or totality of the criterion possessed by the alternatives. If the average or totality changes as the result of addition or deletion of alternatives, then the criteria weights must change (just as has been shown in the supermatrix). Thus, if the supermatrix represents the general case for AHP, it corresponds to Referenced AHP (Table 12) rather than the Distributive mode (Table 10) with assumed independent criteria.

\section{Table 14 -- The Supermatrix, Starting State, with Object 1 Removed}

\begin{tabular}{|c|c|c|c|c|c|c|}
\hline \multirow[b]{2}{*}{ Rox 1} & Box 1 & Box 2 & Box 3 & Object 2 & Object 3 & Object 4 \\
\hline & 0 & 0 & 0 & 0.100 & 0.176 & 0.250 \\
\hline Box 2 & 0 & 0 & 0 & 0.200 & 0.471 & 0.125 \\
\hline Box 3 & $\overline{0}$ & 0 & 0 & 0.700 & 0.353 & 0.625 \\
\hline Object 2 & 0.222 & 0.286 & 0.467 & 0 & 0 & 0 \\
\hline Object 3 & 0.333 & 0.571 & 0.200 & 0 & 0 & 0 \\
\hline Object 4 & 0.444 & 0.143 & 0.333 & 0 & 0 & 0 \\
\hline
\end{tabular}

Table 15 -- The Supermatrix, Stable State, with Object 1 Removed

\begin{tabular}{|c|c|c|c|c|c|c|}
\hline 12 & Box 1 & Box 2 & Box 3 & Object 2 & Object 3 & Object 4 \\
\hline Box 1 & 0 & 0 & 0 & 0.170 & 0.170 & 0.170 \\
\hline Box 2 & $\overline{0}$ & 0 & $\overline{0}$ & 0.264 & 0.264 & 0.264 \\
\hline Box 3 & 0 & 0 & 0 & 0.566 & 0.566 & 0.566 \\
\hline Object 2 & 0.377 & 0.377 & 0.377 & 0 & 0 & 0 \\
\hline Object 3 & 0.321 & 0.321 & 0.321 & 0 & 0 & 0 \\
\hline Object 4 & 0.302 & 0.302 & 0.302 & 0 & 0 & 0 \\
\hline
\end{tabular}

\section{Discussion}

Table 16 summarizes the results of this study. For Vargas's example (1997) with a known composite answer, referenced AHP, linking pin AHP, benchmark measurement, and the supermatrix method all produce correct composite priorities. The distributive mode generated the true baseline priorities, but failed to maintain ratio stability when an alternative was removed. The ideal mode did not generate the correct baseline priorities, but it did maintain the same, albeit incorrect, ratios.

Why does the ideal mode fail to produce correct priorities, why does the distributive mode fail the ratio test upon deletion of an alternative, and why do the other methods produce correct priorities and ratios? The answer can be found in the manner in which criteria priorities are generated.

\section{Table 16 - Summary of Results}

\begin{tabular}{lcccccc} 
& Distributive & Ideal & Referenced & Linking Pin & Benchmark & Supermatrix \\
& mode & Mode & AHP & AHP & AHP & method \\
\hline 1. Generates true priorities & yes & no & yes & yes & yes & yes \\
2. Maintains ratio stability & no & yes & yes & yes & yes & yes \\
\hline
\end{tabular}

Referenced AHP, linking pin AHP, benchmark measurement and the supermatrix method all utilize criteria weights that are dependent upon the alternatives in the choice set. The distributive and ideal modes, on the 
other hand, assume that the criteria priorities are independent of the alternatives. With that assumption, there is no assurance that the initial criteria weights were derived in a manner that will produce true ratios.

In the example used herein, we followed Vargas (1997) and used relative box weights as the criteria weights for the distributive mode. Accordingly, the criteria weights were generated in reference to the alternatives. As seen from Referenced AHP, those criteria weights are the correct ones for a procedure that normalizes the composite weights to sum to one. Had we ignored the underlying alternatives and assumed the criteria were independent of the alternatives, it is likely that we would have produced a different set of weights and composite priorities that do not emulate the true priorities. That is what happened when Object 1 was deleted. We assumed the previous criteria weights were appropriate when they were not. As seen from Referenced AHP, criteria weights must be reassessed whenever the choice set changes.

The ideal mode, which also assumes criteria to be independent of alternatives, produced incorrect initial results because no attempt was made to derive correct criteria weights. Instead, we simply accepted the same criteria weights as used for the distributive mode. As shown by Schoner et al (1993) and the linking pin example, there is a necessary link between the normalization process and the type of questions used to derive criteria weights. Since the ideal mode gives $100 \%$ of the criterion weight to the best alternative, the criteria weights should be derived with the best alternatives kept in mind when making criteria comparisons. The failure to recognize this principle makes the ideal answer problematic and suspect, as has been shown in this study.

The supermatrix method of AHP is the general case that can handle situations of both dependence and independence. Accordingly, we should be able to look upon the supermatrix result to give the correct answer, which it does. The fact that the distributive and ideal modes fail to reproduce the supermatrix results indicates that something is amiss with those methods. It is proposed that criteria weights for the distributive and ideal modes should never be the same and should be derived with the referent alternatives in mind as is done with Referenced and Linking pin AHP. If any alternatives are later added or deleted, then those criteria weights should be reassessed. If the decision maker decides that the weights should not change, then we would have the situation where the criteria are independent of the alternatives. If the reassessment results in changed criteria priorities, then we would have the situation of dependence. The choice is left to the decision maker, but based upon more precise questions that highlight possible dependence. The important point to make is that it is better to assume dependence and then find independence, than to assume independence and fail to find dependence.

Are the defects in the distributive and ideal modes fatal to their use, particularly when they give different ratios for the same question? For the ideal mode, we think so. It is a method proposed to avoid rank reversals, which it does. But without "linking-type" questions, it is not designed to elicit true composite priorities. Thus, the avoidance of reversals is unimportant when the priorities are wrong. Modification of the ideal mode to a linking pin procedure with the ideal alternatives as links would rectify the error. With that change, the ideal mode would become the special case of linking pins where the ideal alternatives are the referent links.

The distributive mode can be used unaltered if we are very sure that we have a situation of pure independence of criteria from alternatives. Such a situation would exist for a decision situation where fixed resources are to be divided amongst a varying number of alternatives. With the addition or deletion of alternatives, we would expect the fixed pie to be distributed differently to the changed choice set.

Another situation where independence is thought to exist is when criteria priorities are specified before the choice set is known. Alternatives are then evaluated according to the criteria as they are brought forth. While such situations exist, we would suggest that either the criteria weights be re-evaluated later when the alternatives become known, or a benchmark or linking pin procedure be used whereby the prior meaning of criteria weights is made more explicit. 


\section{Conclusion}

The six methods illustrated herein each use a different aggregation procedure. What has been ignored or misunderstood by many decision makers and researchers is that criteria weights in different aggregation rules have different interpretations (Choo et al, 1999). The failure to recognize this fact when eliciting the criteria weights can result in composite results that are either incorrect (the ideal mode) or incapable of maintaining ratio stability (distributive mode).

Recognition of the need for congruence between criteria questions and the aggregation process is the point often overlooked in the rank reversal debate. As well, the decision situation is another factor that is overlooked or confused. By paying greater attention to the aggregation process and the decision situation, decision makers will begin to use the available decision tools in a more appropriate manner.

\section{References}

Choo, E. U., Schoner, B. and Wedley, W. C. (1999) "Interpretation of Criteria Weights in Multicriteria Decision Making, "Computers and Industrial Engineering Journal”, 37, 527-541.

Forman, E. H. (1993) Facts and fictions about the Analytic Hierarchy Process, Mathematical and Computer Modelling, 17, 4/5, 19-26.1

Hauser, D. and Peniwati, K. (1996) "On the proper interpretation and impact of the AHP axiom of independence in single and multiple crieeria problems" Journal of Multi-Criteria Decision Analysis, 5.

Saaty, T.L. and L.G. Vargas (1991) The Logic of Priorities, Vol. III, AHP Series, RWS Publications

Saaty, T. L. (1977) “A Scaling Method for Priorities in Hierarchical Structures,” Journal of Mathematical Psychology, 15, 3, 234-81.

Schoner, B. and Wedley, W. C. (1989). Ambiguous Criteria Weights in AHP: Consequences and Solutions, Decision Sciences, 20, 3, 462-475.

Schoner, B., Wedley, W. C. and Choo E. U. (1993) "A unified approach to AHP with linking pins" European Journal of Operations Research, 64,384-392.

Vargas, Luis G. (1997) "Comments on Barzilai and Lootsma Why the Multiplicative AHP is Invalid: A Practical Counterexample”, Journal of Multi-Criteria Decision Analysis, 6, 169-170.

Wedley, W. C., Choo, E. U. and Schoner, B. (1996) "Benchmark Measurement: Between Relative and Absolute", Proceedings of the Fourth International Symposium on the Analytic Hierarchy Process, Burnaby, B. C. Canada, 335-345.

Wedley, W. C., Schoner, B. and Choo, E. U. (1993) "Clustering, dependence and ratio scales in AHP: rank reversals and incorrect priorities with a single criterion" Journal of Multi-Criteria Decision Analysis, $2,145-158$.

Wedley, W. C.; Schoner, B. and Choo, E. U. (1996) "Clusters, Criteria and Calibration -- a Reply to Hauser and Peniwati" Journal of Multi-Criteria Decision Analysis, 5, 267-270. 